\title{
Back to the Roots
}

\section{Mehr und mehr Unternehmen veröffentlichen Nachhaltigkeitsberichte, die sich an einem diffusen Nachhaltigkeitsbegriff orientieren. Ökologische Informationen werden, meist ganz pragmatisch, um soziale und wirtschaftliche Aspekte er- gänzt. So werden zwar die Kriterien verschiedener Ratings und Ranking erfüllt, eine tiefere Reflexion über den Zusammenhang von Produktion, Zukunftsfähig- keit und Gerechtigkeit findet meist jedoch nicht statt. Helfen kann hier nur die Rückbesinnung auf die ursprüngliche Nachhaltigkeitsidee.}

$\mathrm{N}$ Von Ellen Frings achhaltigkeitsberichte sind erst glaubwürdig, wenn sie sich substantiell mit dem Leitbild der nachhaltigen Entwicklung befassen. Reicht dazu aber die schematische Behandlung von Ökologie, Ökonomie und Soziales aus? Oder weisen die Berichte nicht vielmehr darauf hin, dass eine kritische Reflexion des üblichen „DreiSäulen-Modells" der Nachhaltigkeit und eine Rückbesinnung auf die zentralen Ziele einer nachhaltigen Entwicklung anstehen?

Mit diesen Fragen befasste sich das Forschungsvorhaben „Vom Umwelt- zum Nachhaltigkeitsbericht". Das Ergebnis ist ein Leitfaden, der deutlich andere Schwerpunkte setzt als viele andere Leitfäden und Kriterienlisten (1).

\section{Variationen eines Leitbilds}

Um einen Rahmen zur Operationalisierung des Nachhaltigkeitsleitbildes für Unternehmen zu erarbeiten, systematisierte das IFEU zunächst die Entwicklungsstufen der Nachhaltigkeitsdebatte:

- Der Brundtlandbericht der Weltkommission für Umwelt und Entwicklung, ein Basisdokument der Nachhaltigkeitsdebatte, fokussiert auf den verantwortungsvollen Umgang und die gerechte Verteilung der natürlichen Ressourcen. Lösungsansätze sah man in der wirtschaftlichen Entwicklung der ärmeren Länder sowie in neuen ressourcenschonenden Konsum- und Produktionsmustern.

- Die Rio-Konferenz für Umwelt und Entwicklung im Jahr 1992 einen neuen wichtigen Impuls: Umwelt- und Entwicklungsziele seien nur erreichbar sind, wenn ökologische, soziale und wirtschaftliche Zusammenhänge stärker beachtet werden.

- In den folgenden Jahren jedoch änderte sich die Sprachregelung: Zunehmend war von einer Gleichwertigkeit der „drei Säulen“ Ökologie, Ökonomie und Soziales die Rede. Damit wurden die Themen zum Stichwort Nachhaltigkeit immer beliebiger, gelegentlich wurden sogar rein betriebswirtschaftliche Interessen als Nachhaltigkeitsziele deklariert.

- Eine weitere Interpretation des Leitbildes definierte nachhaltige Entwicklung als ein Konzept, das unterschiedliche Interessen gegeneinander abwägt. Damit wurde nachhaltige Entwicklung zu einem Rahmen für Einzelinteressen - und abhängig von den zeitlichen bzw. finanziellen Ressourcen der Verhandlungspartner.

- Erst der Weltgipfel für Umwelt und Entwicklung in Johannesburg, setzte wieder deutlichere Akzente und stellte die Bekämpfung der Armut sowie den Umwelt- und Ressourcenschutz in den Vordergrund.

Das Forschungsvorhaben fasste die Ziele für das unternehmensbezogene Nachhaltigkeitskonzept deutlich enger als andere Ansätze. Ausgehend vom Brundtlandbericht wurden die Umwelt- und Ressourcenschonung sowie die internationale Verteilungsgerechtigkeit in den Vordergrund gerückt. Soziale und wirtschaftliche Aspekte erhielten darüber hinaus in dem Maß Beachtung, wie sie

- der Entwicklung ärmerer Länder beziehungsweise der weltweiten Verteilungsgerechtigkeit dienen und

- eine breite Akzeptanz für eine Politik in den Industrieländern fördern, die sich an umweltund entwicklungspolitischen Zielen orientiert.

Akzeptanz für eine solche Politik ist um so schwieriger zu erreichen, je stärker soziale und wirtschaftliche Probleme die öffentliche Diskussion dominieren. Daher wurden auch drängende soziale Themen in den Industrieländern, wie Arbeitslosigkeit, in das Zielsystem integriert. Gleichzeitig braucht nachhaltige Entwicklung ein hohes Maß an sozialer Teilnahme und Verantwortlichkeit für das eigene Handeln.
Die Entwicklung sozialer Kompetenzen wurde daher als weitere relevante soziale Zielebene definiert.

\section{Ein neves - altes Konzept}

Auf dieser Grundlage wertete das IFEU 14 Kriterienkataloge für Nachhaltigkeitsberichte, Ratings und Rankings sowie Managementinstrumente aus. Fazit: Viele soziale und ökonomische Kriterien zielen auf die Entwicklung einer höheren Lebensqualität im eigenen Umfeld, haben aber keinen Bezug zu Ressourcenschutz und Verteilungsgerechtigkeit. Die Konzepte wurden daher gefiltert und die ausgewählten Kriterien in einen neuen Anforderungskatalog aufgenommen.

Ergänzend untersuchte das IFEU die externen Anforderungen an Unternehmen und ihre Nachhaltigkeitsberichte. Seit einigen Jahren tritt hier eine Anspruchsgruppe verstärkt auf den Plan: die Globalisierungskritiker. In multinationalen Unternehmen sehen sie treibende Kräfte der Globalisierung und damit Mitverantwortliche für ihre Folgen. Auf der Negativliste stehen Öko- und Sozialdumping, wettbewerbsbeschränkende Praktiken, die Dominierung der Politik durch die Wirtschaft sowie der zunehmende Abbau von Arbeitskräften mit der Folge einer immer größeren Kluft zwischen Arm und Reich. Im Rahmen des Projektes wurden die Positionen der Globalisierungskritiker ausgewertet und in den Anforderungskatalog integriert. Eine weitere Grundlage für den Leitfaden waren Interviews mit 20 Expertinnen und Experten aus Umwelt- und Entwicklungsverbänden, Politik und Gewerkschaften, Umwelt- und Wirtschaftswissenschaften, Arbeitsmarktforschung sowie Behörden. Viele Interviewpartner hoben hervor, dass Nachhaltigkeitsberichte in erster Linie die Geschäftstätigkeiten der Unternehmen und ihre Auswirkungen auf das Unternehmensumfeld reflektieren und dabei an aktuellen politischen, wirtschaftlichen und gesellschaftlichen Entwicklungen anknüpfen sollten. Explizit wurden Themen wie Globalisierung, Armut und Entwicklung, die Wettbewerbsdebatte und die Ressourcenverknappung genannt. Die geplante Ablösung vom Drei-SäulenModell wurde durchgängig begrüßt.

Aus den verschiedenen Analyseschritten wurde ein Anforderungskatalog, orientiert an sechs Handlungsfeldern von Unternehmen, abgeleitet und in einem Workshop mit 30 Vertretern aus Unternehmen, umwelt- und entwicklungspolitischen Gruppen, Behörden, Wissenschaft und Beratung abgestimmt (Tabelle 1). 


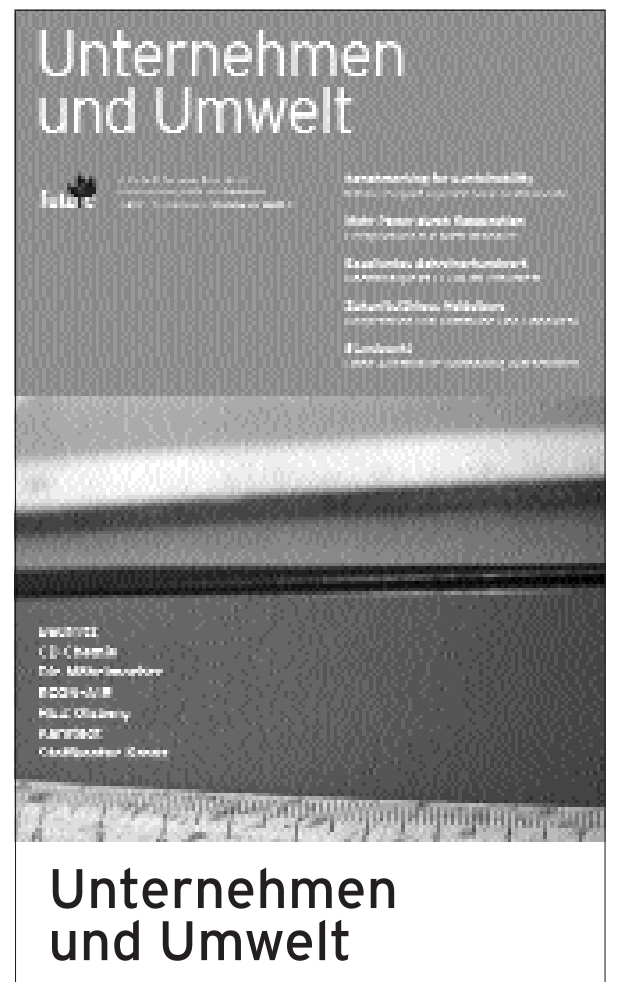

. ist die Zeitschrift für umweltorientierte Unternehmenspolitik von future e.V.

. bietet Fachinformationen zum Umweltschutz und Best-Practice-Beispiele aus Unternehmen.

.. bietet viermal im Jahr Unternehmensbeispiele, Literatur- und Veranstaltungshinweise.

Mit einem Schwerpunktthema in jedem Heft. Zum Beispiel:

3-4/02: Benchmarking for Sustainability 2/02: Ökomarketing

1/02: Nachhaltigkeit und Gewerbebau

4/01: Nachhaltigkeit im Handwerk

3/01: Klimaschutz

2/01: Nachhaltiges Investment

1/01: Grüne Bauwirtschaft

4/00: Kooperative Textilwirtschaft

3/00: Integrierte Produktpolitik

2/00: Soft Facts im Umweltschutz

Bestellen Sie ihr kostenloses Probe-Abo im Internet unter

w w w. f u t u re - e v.de

future e.V. Büro Bochum

Am Varenholt 123

44797 Bochum

Fon: 0234/9799513

Fax: 0234/9799514

bochum@future-ev.de

www.future-ev.de

Tab. 1: Nachhaltigkeitsziele in unternehmerischen Handlungsfeldern

Unternehmen als gestaltende Wirtschaftskraft

- Gerechte internationale Verteilung von Wohlstand und Wertschöpfung

- Faire Marktregeln in der Wertschöpfungskette u.a. Wirtschaftsbeziehungen

- Ausgewogene, beschäftigungsfördernde Wirtschaftsstrukturen

\section{Unternehmen als Anbieter von Produkten und Dienstleistungen}

- Ressourceneffiziente Erfüllung gesellschaftlicher Bedürfnisse

- Information und Marketing für ökologische Konsummuster

- Übernahme von Produktverantwortung

Unternehmen als Transformator von Stoffen und Energien
- Ökologische Optimierung der Produktionsprozesse
Unternehmen als Ort der Beschäftigung und des Lernens
- Existenzsicherung durch Beschäftigung und Qualifizierung
- Werte vermitteln
Unternehmen als Auslöser von Struktur- und Regionalentwicklung
- Ausgewogene, umweltverträgliche Regionalentwicklung
- Strukturentwicklung in Entwicklungs- und Schwellenländern
Unternehmen als politische Interessensvertreter
- Unterstützung nachhaltigkeitsorientierter Politik
- lebendiger gesellschaftlicher Dialog über nachhaltige Entwicklung

\section{Der Leiffaden}

Ergebnis des Projektes ist die 70-seitige Broschüre „Zukunftsfähiges Wirtschaften - Ein Leitfaden zur Nachhaltigkeitsberichterstattung von Unternehmen“ (2). Zielgruppe sind in erster Linie Großunternehmen, aber auch kleinere Unternehmen finden zahlreiche Anknüpfungspunkte für ihr Nachhaltigkeitsengagement.

Ausgehend von der aktuellen Nachhaltigkeitsund Globalisierungsdebatte lotet der Leitfaden zunächst die Handlungsspielräume der Unternehmen bei wachsendem Wettbewerbsdruck aus und skizziert die Doppelrolle der Global Player zwischen Aktion und Reaktion in einem sich zunehmend verändernden wirtschaftlichen Umfeld. Im darauf folgenden Praxisteil erhalten die Unternehmen Anleitungen, wie sie die interne Auseinandersetzung um nachhaltige Entwicklung organisieren können. Der Leitfaden unterstiutzt die Unternehmen dabei, eigene Prioritäten zu setzen. Fragelisten zu den Handlungsfeldern helfen, die Inhalte weiter zu konkretisieren und individuelle Kommunikationsstrategien via Bericht, Internet und andere Kommunikationskanäle zu erarbeiten. Durch einen Check zur Selbsteinstufung können die Unternehmen ihren momentanen Status bestimmen und „,blinde Flecken“ im Nachhaltigkeitsengagement identifizieren. Ein umfassender Anhang liefert Quellen und Kurzbeschreibungen zu Nachhaltigkeitskonzepten für Unternehmen sowie zu Umwelt- und Sozialstandards beziehungsweise Verhaltenskodizes.
Mit seinem prozessorientierten Ansatz stellt der Leitfaden eine gute Ergänzung zu den „Sustainability Reporting Guidelines" der Global Reporting Initiative (GRI) dar. Die in einem weltweiten Abstimmungsprozess erarbeiteten Kennzahlen des GRI-Leitfadens könnten ein einheitliche Datengerüst bieten und einen Vergleich zwischen den berichtenden Unternehmen ermöglichen. Vor allem in ihrer Kombination können beide Leitfäden international eingebundene Unternehmen zukünftig bei ihrer Nachhaltigkeitsberichterstattung unterstützen.

\section{Anmerkungen}

(1) Im Auftrag des Landes Baden-Württemberg untersuchte das Institut für Energie- und Umwelfforschung (IFEU) den Stand der Nachhaltigkeitsdiskussion und die Anforderungen der Stakeholder. Vergleiche: Frings, E.: Vom Umwelt- zum Nachhaltigkeitsbericht - Anforderungen und Kriterien. Abschlussbericht, 2003. Download: www.bwplus.fzk.de/berichte/SBer/BWA20010SBer.pdf

(2) Bezug des Leiffadens "Zukunftsfähiges Wirtschaften" beim Ministerium für Umwelt und Verkehr, Kernerplatz 9 , 70182 Stuttgart.

\section{Die Autorin}

Ellen Frings ist Fachbereichsleiterin für Umweltmanagement am Institut für Energie- und Umweltforschung (IFEU).

Kontakt: IFEU, Wilckensstr. 3, 69120 Heidelberg, Tel.06221-476756, E-Mail: ellen.frings@ifeu.de 
(c) 20I0 Authors; licensee IÖW and oekom verlag. This is an article distributed under the terms of the Creative Commons Attribution Non-Commercial No Derivates License (http://creativecommons.org/licenses/by-nc-nd/3.o/), which permits unrestricted use, distribution, and reproduction in any medium, provided the original work is properly cited. 